WUGRAV-95-6

\title{
Statistics of the Microwave Background Anisotropies Caused by the Squeezed Cosmological Perturbations
}

\author{
L. P. Grishchuk* \\ McDonnell Center for the Space Sciences, Physics Department \\ Washington University, St. Louis, Missouri 63130 \\ and \\ Sternberg Astronomical Institute, Moscow University \\ 119899 Moscow, V234, Russia \\ (June 20, 2022 \\ Submitted to Physical Review D)
}

\begin{abstract}
It is likely that the observed large-angular-scale anisotropies in the microwave background radiation are induced by the cosmological perturbations of quantum-mechanical origin. Such perturbations are now placed in squeezed vacuum quantum states and, hence, are characterized by large variances of their amplitude. The statistical properties of the anisotropies should reflect the underlying statistics of the squeezed vacuum quantum states. The theoretical variances for the temperature angular correlation function are derived and described quantitatively. It is shown that they are indeed large. Unfortunately, these large theoretical statistical uncertainties will make the extraction of cosmological information from the measured anisotropies a much more difficult problem than we wanted it to be.
\end{abstract}

PACS numbers: 98.80.Cq. 04.30.-w, 42.50.Dv, 98.70.Vc

*grishchu@howdy.wustl.edu 


\section{INTRODUCTION}

The line of reasoning in this paper is as follows.

We see the anisotropies in the microwave background radiation at the largest angular scales [1]. Observers convincingly argue that this is a genuine cosmological effect.

If the large-angular-scale anisotropy in the microwave background is really produced by cosmological perturbations (density perturbations, rotational perturbations, gravitational waves), then their today's wavelengths are of the order and longer than today's Hubble radius $l_{H}$. Strictly speaking, all wavelengths give contributions to the anisotropy at every given angular scale. But if the spectrum of the perturbations is not excessively "red" or "blue", the dominant contribution is provided by wavelengths indicated above. In fact, the major contribution to the quadrupole anisotropy is provided by wavelengths somewhat longer than $l_{H}$.

In the expanding Universe, the wavelengths of perturbations increase in proportion to the cosmological scale factor. The wavelengths that are longer than some length scale today have always been longer than that scale in the past. Moreover, the wavelengths of the perturbations of our interest are much longer than the Hubble radius defined at the previous times, when one goes back in time up to the era of primordial nucleosynthesis - the earliest era of which we have observational data. It is hard to imagine (although it does not seem to be logically impossible) that cosmological perturbations of our interest, with such long wavelengths, could have been generated by local physical processes during the interval of time between the era of primordial nucleosynthesis and now. We are bound to conclude that these perturbations were generated in the very early Universe, before the era of primordial nucleosynthesis. There is still 80 orders of magnitude, in terms of energy density, to go from the era of primordial nucleosynthesis to the Planck era; a lot of things could have happened in between.

The law of evolution of the very early Universe is not known, but it is likely that it could have been significantly different from the law of expansion of the radiation-dominated Universe. If so, some amount of cosmological perturbations must have been generated quantum-mechanically, as a result of parametric interaction of the quantized perturbations with strong variable gravitational field of the very early Universe. Gravitational waves have been generated inevitably, while density and rotational perturbations - if we were lucky; see [2] and references therein. (If the cosmological scale factor has always been the one of the radiation-dominated Universe, we must stop here, because the parametric coupling vanishes in this case, and cosmological perturbations cannot be amplified classically and cannot be generated quantum-mechanically.) The amount and spectrum of the generated perturbations depend on the law of evolution of the very early Universe (the strength and variability of the gravitational pump field), and this is how we can learn about what was going on there. In particular, the law of evolution of the very early Universe could have been of inflationary type.

If the cosmological perturbations were generated quantum-mechanically, they should now be placed in the squeezed vacuum quantum states [3] (for an introduction to squeezed states see, for example, Ref. [4,5] and the pioneering works quoted there). Squeezing of cosmological perturbations might have degraded by now at short wavelengths but should survive at long wavelengths, especially in the case of gravitational waves. 
Now, the squeezed vacuum quantum states can only be squeezed in the variances of phase which unavoidably means the increased variances in amplitude. The statistical properties of the squeezed vacuum quantum states are significantly different from the statistical properties of the "most classical" quantum states - coherent states. This is well illustrated by the fact that the variance of the number of quanta in a strongly squeezed vacuum quantum state is much larger than the variance of the number of quanta in the coherent state with the same mean number of quanta $\langle N\rangle,\langle N\rangle \gg 1$. For a squeezed vacuum state the variance is $\left\langle N^{2}\right\rangle$ $-\langle N\rangle^{2}=2\langle N\rangle(\langle N\rangle+1)$, while for a coherent state it is $\left\langle N^{2}\right\rangle-\langle N\rangle^{2}=\langle N\rangle$.

The statistical properties of squeezed cosmological perturbations will inevitably be reflected in statistical properties of the microwave background anisotropies caused by them. Squeezing is a phase-sensitive phenomenon, and to fully extract its properties the quantum optics experimenters use the phase-sensitive detecting techniques based on a local oscillator. In cosmology, we are very far from being able to build a local oscillator, except of maybe, in the distant future, for short gravitational waves. Besides, in our study of the microwave background anisotropies, we are interested in so long-wavelength perturbations that it would take billions of years to wait for seeing the time dependent oscillations of variances in the quadrature components of the perturbation field. On the other hand, the amount of cosmological squeezing is enormously greater than what is achieved in quantum optics laboratory experiments. In cosmology, we can only rely on the phase-insensitive, direct detection. One can expect that the underlying large variances of the amplitude of cosmological perturbations should result in large statistical deviations from the mean values for the microwave anisotropies.

A detailed study and proof of this statement is the purpose of this paper.

At this point it is necessarry to say that the quantum-mechanical generating mechanism has become popular in the context of the inflationary hypothesis. However, the inflationary literature associates the explanation of the phenomenon with such things as ambiguity in the choice of time in the De Sitter universe, temperature, tremendous inflation of scales, and so on. The basic concepts are adjusted accordingly. Instead of amplification, with the emphasis on a nonvanishing parametric coupling, increase of amplitude at the expense of energy of the pump field, quantum-mechanical generation of waves (particles) in strictly correlated pairs, etc., inflationary literature speaks about magnification, with the emphasis on "stretching the waves" and "crossing the horizons". Inflationists did not get puzzled with their "standard" formula for density perturbations which states that one can produce arbitrarily large amount of density perturbations by practically doing nothing.

The "standard" formula relates the amplitude of density perturbations today with the values of the scalar field during inflation. Let us consider, for definiteness, perturbations of the matter density $\delta \rho / \rho$ with today's wavelengths of the order of today's Hubble radius $l_{H}$. The "standard" formula says that

$$
\left.\frac{\delta \rho}{\rho}\right|_{H} \sim \frac{H^{2}}{\dot{\phi}\left(t_{1}\right)}
$$

where the right hand side of this formula is supposed to be evaluated at the time $t_{1}$ when the wavelengths of our interest were "crossing the horizon" during inflation. Let us agree with the so-called "slow-roll" approximation and assume that the Hubble parameter $H$ was almost constant during that epoch, $\dot{H} \ll H^{2}$. Let us take the numerical value of $H$ during 
that epoch at the level, say, 10 orders of magnitude smaller than the Planck value of $H$. For quantum-mechanically generated gravitational waves, it would result in the today's amplitude $h \approx 10^{-10}$ and the induced anisotropies of the microwave background $\delta T / T \approx 10^{-10}$ which are much lower than what is currently discussed in the experiment. However, for density perturbations, according to the "standard" formula, the situation is totally different. Without changing anything in the curvature of the space-time responsible for the generating process (that is, leaving $H$ almost constant and at the same numerical level), but simply sending $\dot{\phi}\left(t_{1}\right)$ to zero (which corresponds, due to Einstein equations, to sending $\dot{H}$ to zero, i.e., making the "slow-roll" approximation better and better, making the expansion law closer and closer to the De Sitter expansion) one produces arbitrarily large $\left.(\delta \rho / \rho)\right|_{H}$. Inflationists love to stress that the De Sitter gravitational pump field generates perturbations with the Harrison-Zeldovich (scale-invariant, flat) spectrum. What they do not stress is that, according to their "standard" formula for density perturbations, the amplitudes of the scale-invariant spectrum are infinite, and the amplitudes of the almost scale-invariant spectrum are almost infinite. Instead of blaming their own formula, inflationists blame the scalar field potentials. This formula is the reason for rejecting certain scalar field potentials on the grounds that they generate "too much" of density perturbations, for claims that the contribution of gravitational waves to $\delta T / T$ is "negligibly small" in the limit of the De Sitter expansion, and even for claims about copious production of black holes during inflation. The "standard" formula has been recently reiterated [6]. It has been formulated, essentially, as the following "standard result": "... we see that the scalar perturbations can be very strongly amplified" [the increase of numerical value from (almost) zero to (almost) infinity] "in the course of the transition" [the instantaneous change of the cosmological scale factor from one power-law behavior to another power-law behavior]. The authors of the paper [6] assure the trusting reader: "We think that there is nothing strange about this ...".

The "standard" inflationary formula is unacceptable physically, and it does not come as a surprise that it is incorrect mathematically. It may follow from reach imagination, but it does not follow from equations. (For technical details see $[2,7]$ especially Exercises 1, 2, 3 in Ref. [7].) According to the calculations of Ref. [2], the contribution of the quantum-mechanically generated gravitational waves to the large-angular-scale anisotropy is greater (even in the limit of the De Sitter expansion) than the contribution of the quantummechanically generated density perturbations. However, in this paper, we will not discuss any longer the relative contributions to $\delta T / T$ supplied by cosmological perturbations of different nature (density perturbations, rotational perturbations, or gravitational waves). We will concentrate on the consequences of their common origin - quantum mechanics and squeezing. Our discussion will be equally well applicable to the perturbations of any nature if they have the same origin. 


\section{THE GENERAL EQUATIONS FOR QUANTIZED COSMOLOGICAL PERTURBATIONS}

Here we will briefly summarize some basic information about quantized cosmological perturbations (see $[2,7]$ and references therein).

The metric of the homogeneous isotropic universe can be written in the form

$$
d s^{2}=-a^{2}(\eta)\left(d \eta^{2}-\gamma_{i j} d x^{i} d x^{j}\right)
$$

where $\gamma_{i j}$ is the spatial metric. For reasons of simplicity, we will be considering only spatially flat universes, that is $\gamma_{i j}=\delta_{i j}$.

Following Lifshitz, it is convenient to write the perturbed metric in the form

$$
d s^{2}=-a^{2}(\eta)\left[d \eta^{2}-\left(\delta_{i j}+h_{i j}\right) d x^{i} d x^{j}\right],
$$

where $h_{i j}$ are functions of $\eta$-time and spatial coordinates. By writing the perturbed metric in this form we do not lose anything in the physical content of the problem, but we gain considerably in the mathematical tractability of the perturbed Einstein equations. The one who is interested in solving equations (not just in discussing them) will certainly be interested in a simpler form of equations, even if only as "an insurance against mistakes" (the term is borrowed from [6]) when searching for solutions. Those who prefer "gaugeinvariant formalisms" are welcome to take the found solution and compute with its help whichever "gauge-invariant quantity" they like. These quantities, being "gauge-invariant", have the same values in all "gauges".

The components $h_{i j}$ of the perturbed gravitational field can be classified in terms of scalar, vector, and tensor eigenfunctions of the Laplace differential operator. The components of the perturbed energy-momentum tensor can also be classified in the same manner. After that, the linearized Einstein equations reduce to a set of ordinary differential equations, separately for scalar (density perturbations), vector (rotational perturbations), and tensor (gravitational waves) parts.

The number of independent unknown functions of time that can potentially be present (on grounds of the classification scheme) in the perturbed Einstein equations is always greater than the number of independent equations. It is 6 functions and 4 equations for density perturbations, 3 functions and 2 equations for rotational perturbations, and 2 functions and 1 equation for gravitational waves. In order to make the system of equations closed, it is necessary to say something about the perturbed components of the energy-momentum tensor or to specify from the very beginning the form of the energy-momentum tensor. The popular choices are perfect fluids and scalar fields. Even for gravitational waves, it is not a totally trivial question what their definition is (see, for example, Ref. [8]). However, after everything is being set, and as soon as the scale factor $a(\eta)$ (the background solution) is known, the general solution to the perturbed equations can be found. In practice, exact solutions are being found piecewise, at the intervals of evolution where the energy-momentum tensor has simple prescribed forms.

We can now write the quantum-mechanical operator for the perturbations of the gravitational field $h_{i j}$ in the following universal form:

$$
h_{i j}=\frac{C}{a(\eta)} \frac{1}{(2 \pi)^{3 / 2}} \int_{-\infty}^{\infty} d^{3} n \sum_{s=1}^{2} \stackrel{s}{p}_{i j}(\mathbf{n}) \frac{1}{\sqrt{2 n}}\left[\stackrel{s}{c}_{\mathbf{n}}(\eta) e^{i \mathbf{n x}}+\stackrel{s}{c}_{\mathbf{n}}^{\dagger}(\eta) e^{-i \mathbf{n} \mathbf{x}}\right] .
$$


We will start the explanation of Eq. (3) from the polarization tensors $\stackrel{s}{p}_{i j}$. Let us introduce, in addition to the unit wave-vector $\mathbf{n} / n$, two more unit vectors $l_{i}, m_{i}$, orthogonal to each other and to $\mathbf{n}$ :

$$
\begin{aligned}
\frac{n_{i}}{n} & =(\sin \theta \cos \phi, \sin \theta \sin \phi, \cos \theta), \quad l_{i}=(\sin \phi,-\cos \phi, 0), \\
m_{i} & = \pm(\cos \theta \cos \phi, \cos \theta \sin \phi,-\sin \theta),
\end{aligned}
$$

+ for $\theta<\frac{\pi}{2}, \quad-$ for $\theta>\frac{\pi}{2}$.

The two independent polarization tensors, $s=1,2$, for each class of perturbations, can be written as follows. For gravitational waves:

$$
\stackrel{1}{p}_{i j}(\mathbf{n})=\left(l_{i} l_{j}-m_{i} m_{j}\right), \quad \stackrel{2}{p}_{i j}(\mathbf{n})=\left(l_{i} m_{j}+l_{j} m_{i}\right)
$$

For rotational perturbations:

$$
\stackrel{1}{p}_{i j}(\mathbf{n})=\frac{1}{n}\left(l_{i} n_{j}+l_{j} n_{i}\right), \quad \stackrel{2}{p}_{i j}(\mathbf{n})=\frac{1}{n}\left(m_{i} n_{j}+m_{j} n_{i}\right)
$$

For density perturbations:

$$
\stackrel{1}{p}_{i j}(\mathbf{n})=\sqrt{\frac{2}{3}} \delta_{i j}, \quad \stackrel{2}{p}_{i j}(\mathbf{n})=-\sqrt{3} \frac{n_{i} n_{j}}{n^{2}}+\frac{1}{\sqrt{3}} \delta_{i j}
$$

The polarization tensors of each class satisfy the conditions $\stackrel{\stackrel{s}{p}}{p_{i j}} \stackrel{s^{\prime}}{p^{i j}}=2 \delta_{s s^{\prime}}, \stackrel{s}{p}$ ij $(-\mathbf{n})=\stackrel{s}{p}$ ij $(\mathbf{n})$. In practical handling of the density perturbations it proves convenient to use sometimes, in addition to the scalar polarization component $\stackrel{1}{p}_{i j}$, the longitudinal-longitudinal component (proportional to $n_{i} n_{j}$ ) instead of $\stackrel{2}{p}_{i j}$. The explicit functional dependence of the polarization tensors is needed for the calculation of various angular correlation functions.

The evolution of the creation and annihilation operators $\stackrel{s}{c_{\mathbf{n}}}(\eta), \stackrel{s}{c}_{\mathbf{n}}^{\dagger}(\eta)$, for each class of perturbations and for each polarization state, is defined by the Heisenberg equations of motion:

$$
\frac{d c_{\mathbf{n}}(\eta)}{d \eta}=-i\left[c_{\mathbf{n}}(\eta), H\right], \quad \frac{d c_{\mathbf{n}}^{\dagger}(\eta)}{d \eta}=-i\left[c_{\mathbf{n}}^{\dagger}(\eta), H\right] .
$$

The dynamical content of the problem is determined by the Hamiltonian $H$. Its form depends on the class of perturbations and additional assumptions about the energy-momentum tensor which we have to make, as was discussed above.

Under the simplest assumptions about gravitational waves (waves interact only with the background gravitational field, there is no anisotropic material sources) the Hamiltonian for each polarization component takes on the form

$$
H=n c_{\mathbf{n}}^{\dagger} c_{\mathbf{n}}+n c_{-\mathbf{n}}^{\dagger} c_{-\mathbf{n}}+2 \sigma(\eta) c_{\mathbf{n}}^{\dagger} c_{-\mathbf{n}}^{\dagger}+2 \sigma^{*}(\eta) c_{\mathbf{n}} c_{-\mathbf{n}}
$$

where the coupling function $\sigma(\eta)$ is $\sigma(\eta)=\frac{i}{2} \frac{a^{\prime}}{a}$.

For rotational perturbations, assuming that the primeval matter is capable of supporting torque oscillations, assuming that the oscillations are minimally coupled to gravity, and 
assuming that the torsional velocity of sound is equal to the velocity of light, the Hamiltonian for each polarization component reduces to exactly the same form (6) with the same coupling function $\sigma(\eta)$.

For density perturbations, we consider specifically a minimally coupled scalar field with arbitrary scalar field potential as a model for matter in the very early Universe, and perfect fluids at the later eras. The quantization is based on the scalar polarization component (the function of time responsible for another polarization state is not independent). There is only one independent sort of creation and annihilation operators in this case. The operators $\stackrel{s}{c_{\mathbf{n}}}(\eta), \stackrel{s}{c}_{\mathbf{n}}^{\dagger}(\eta)$ are expressible in terms of the operators $d_{\mathbf{n}}(\eta), d_{\mathbf{n}}^{\dagger}(\eta)$ for which the Hamiltonian has again the same form (6) but with the coupling function $\sigma(\eta)=\frac{i}{2} \frac{(a \sqrt{\gamma})^{\prime}}{a \sqrt{\gamma}}$, where

$$
\gamma(\eta)=1+\left(\frac{a}{a^{\prime}}\right)^{\prime}
$$

For density perturbations, it is the operators $d_{\mathbf{n}}(\eta), d_{\mathbf{n}}^{\dagger}(\eta)$ that participate in Eqs. (5), (6).

Now, let us turn to the constant $C$ in Eq. (3). Its value is determined by the normalization of the field of each class to the "half of the quantum in each mode". Under the assumptions listed above, one derives $C=\sqrt{16 \pi} l_{P l}$ for gravitational waves, $C=\sqrt{32 \pi} l_{P l}$ for rotational perturbations, and $C=\sqrt{24 \pi} l_{P l}$ for density perturbations, where $l_{P l}$ is the Planck length, $l_{P l}=\left(G \hbar / c^{3}\right)^{1 / 2}$.

The form of the Hamiltonian (6) dictates the form of the solution (Bogoliubov transformation) to Eq. (5):

$$
\begin{aligned}
& c_{\mathbf{n}}(\eta)=u_{n}(\eta) c_{\mathbf{n}}(0)+v_{n}(\eta) c_{-\mathbf{n}}^{\dagger}(0) \\
& c_{\mathbf{n}}^{\dagger}(\eta)=u_{n}^{*}(\eta) c_{\mathbf{n}}^{\dagger}(0)+v_{n}^{*}(\eta) c_{-\mathbf{n}}(0)
\end{aligned}
$$

where $c_{\mathbf{n}}(0), c_{\mathbf{n}}^{\dagger}(0)$ are the initial values of the operators taken long before the interaction with the pump field became important $(\sigma(\eta) / n \rightarrow 0)$ and which define the vacuum state $c_{\mathbf{n}}(0)|0\rangle=0$. The complex functions $u_{n}(\eta), v_{n}(\eta)$ obey coupled first-order differential equations following from Eq. (4) and satisfy the condition $\left|u_{n}\right|^{2}-\left|v_{n}\right|^{2}=1$ which guarantees that the commutator relationship $\left[c_{\mathbf{n}}(0), c_{\mathbf{m}}^{\dagger}(0)\right]=\delta^{3}(\mathbf{n}-\mathbf{m})$ is satisfied at all times, $\left[c_{\mathbf{n}}(\eta), c_{\mathbf{m}}^{\dagger}(\eta)\right]=\delta^{3}(\mathbf{n}-\mathbf{m})$. If one introduces the function $\mu_{n}(\eta)=u_{n}(\eta)+v_{n}^{*}(\eta)$, one recovers from the equations for $u_{n}(\eta), v_{n}(\eta)$ the classical equations of motion. For gravitational waves:

$$
\mu_{n}^{\prime \prime}+\left[n^{2}-\frac{a^{\prime \prime}}{a}\right] \mu_{n}=0
$$

For rotational perturbations:

$$
\mu_{n}^{\prime \prime}+\left[n^{2} \frac{v_{t}^{2}}{c^{2}}-\frac{a^{\prime \prime}}{a}\right] \mu_{n}=0 .
$$

where $v_{t}$ is the torsional velocity of sound which we assumed above to be $c$. For the scalar field density perturbations:

$$
\mu_{n}^{\prime \prime}+\left[n^{2}-\frac{(a \sqrt{\gamma})^{\prime \prime}}{a \sqrt{\gamma}}\right] \mu_{n}=0
$$


In the Schrödinger picture, the initial vacuum quantum state $\left|0_{\mathbf{n}}\right\rangle\left|0_{-\mathbf{n}}\right\rangle$ evolves into a two-mode squeezed vacuum quantum state. In our problem, each of the two-mode squeezed vacuum quantum states is a product of two identical one-mode squeezed vacuum quantum states which correspond to the decomposition of the real field $h_{i j}$ over real spatial harmonics $\sin \mathbf{n x}$ and $\cos \mathbf{n x}$. In the Heisenberg picture, the initial vacuum quantum state does not evolve in time and is the same now.

By using Eq. (7) one can present the field (3) in the form

$$
h_{i j}(\eta, \mathbf{x})=C \frac{1}{(2 \pi)^{3 / 2}} \int_{-\infty}^{\infty} d^{3} n \sum_{s=1}^{2} \stackrel{s}{p}_{i j}(\mathbf{n}) \frac{1}{\sqrt{2 n}}\left[{ }_{h}^{s}(\eta) e^{i \mathbf{n x}}{ }_{C_{\mathbf{n}}}^{s}(0)+\stackrel{s}{h}_{n}^{*}(\eta) e^{\left.-i \mathbf{n x}{ }_{C_{\mathbf{n}}}^{s^{\dagger}}(0)\right],}\right.
$$

where the functions $\stackrel{s}{h}_{n}(\eta)$ are $\stackrel{s}{h}(\eta)=\frac{1}{a(\eta)}\left[\stackrel{s}{u}_{n}(\eta)+\stackrel{s}{v}_{n}^{*}(\eta)\right]$. For gravitational waves and rotational perturbations, the functions $\stackrel{s}{h}$ are simply $\stackrel{s}{h}{ }_{n}=\stackrel{s}{\mu} / a$ where $\stackrel{s}{\mu}_{n}$ are solutions to Eqs. (8), (9) with appropriate initial conditions. For density perturbations, the functions $\stackrel{s}{h_{n}}$ are derivable from solutions to Eq. (10) in accord with the relationship between $c$ and $d$

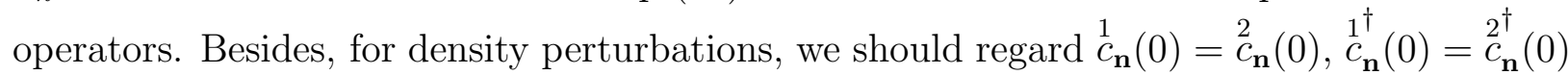
in Eq. (11). In all cases, for a given cosmological model, that is for a model in which the scale factor $a(\eta)$ is known from the very early times and up to now, the functions $\stackrel{s}{h}$ can be found from the classical equations of motion with appropriate initial conditions.

It follows from Eq. (11) that the mean quantum-mechanical value of the field $h_{i j}$ is zero at every spatial point and at every moment of time, $\left\langle 0\left|h_{i j}\right| 0\right\rangle=0$. One can also calculate variances of the field, that is the expectation values of its quadratic combinations. One useful quantity is $h_{i j} h^{i j}$. By manipulating with the product of two expressions (11), using the summation properties of the polarization tensors, and remembering that the only nonvanishing correlation function is

$$
\left\langle 0\left|\stackrel{s}{C_{\mathbf{n}}}(0){ }_{c_{\mathbf{n}^{\prime}}}^{s^{\prime \dagger}}(0)\right| 0\right\rangle=\delta_{s s^{\prime}} \delta^{3}\left(\mathbf{n}-\mathbf{n}^{\prime}\right)
$$

one can derive the formula

$$
\left\langle 0\left|h_{i j}(\eta, \mathbf{x}) h^{i j}(\eta, \mathbf{x})\right| 0\right\rangle=\frac{C^{2}}{2 \pi^{2}} \int_{0}^{\infty} n \sum_{s=1}^{2}\left|h_{n}(\eta)\right|^{2} d n .
$$

Equation (12) shows that the variance is independent of the spatial point $\mathbf{x}$ but does depend on time.

The expression under the integral in formulas such as Eq. (12) is usually called the power spectrum (in this case, it is the power spectrum of the quantity $h_{i j} h^{i j}$ ):

$$
P(n)=\frac{C^{2}}{2 \pi^{2}} n \sum_{s=1}^{2}\left|h_{n}(\eta)\right|^{2}
$$

In cosmology, it is common to use the power spectrum defined in terms of the logarithmic frequency interval, that is the function

$$
P_{Z}(n)=\frac{C^{2}}{2 \pi^{2}} n^{2} \sum_{s=1}^{2}\left|h_{n}(\eta)\right|^{2}
$$


( $Z$ from Zeldovich). We are mostly interested in the power spectrum of cosmological perturbations in the present Universe, at the matter-dominated stage. This spectrum is never smooth as a function of frequency (wave-number) $n$. Squeezing and associated standing wave pattern of the field make the spectrum an oscillating function of $n$ for each moment of time. However, the spectrum is smooth for sufficiently long waves. At a given moment of time, it applies to all perturbations whose wavelengths are of the order and longer than the Hubble radius defined at that time. Moreover, the smooth part of the spectrum is powerlaw dependent on $n$ if the scale factor $a(\eta)$ of the very early Universe (the pump field) was power-law dependent on $\eta$-time.

Let us assume that the scale factor at the initial stage of expansion was

$$
a(\eta)=l_{0}|\eta|^{1+\beta}
$$

where $l_{o}$ and $\beta$ are constants. If the evolution is governed by a scalar field, the Einstein equations require the constant $\beta$ to be $\beta \leq-2$. The value $\beta=-2$ corresponds to the De Sitter expansion. At later times, the scale factor changed to the laws of the radiationdominated and matter-dominated universes. From solutions for $\stackrel{s}{h}(\eta)$ traced up to the matterdominated stage, one can find

$$
\sum_{s=1}^{2}\left|\stackrel{s}{h}_{n}(\eta)\right|^{2} \sim \frac{1}{l_{o}^{2}} n^{2 \beta+2} \quad \text { and } \quad P_{Z}(n) \sim \frac{l_{P l}^{2}}{l_{o}^{2}} n^{2(\beta+2)} .
$$

It is convenient to introduce the characteristic amplitude $h(n)$ of the metric perturbations defining this amplitude as the standard deviation (square root of variance) of the perturbed gravitational field per logarithmic frequency interval. In the long-wavelength limit under discussion, this quantity is universally expressed (both, for gravitational waves and density perturbations) by the formula [20]

$$
h(n) \sim \frac{l_{P l}}{l_{o}} n^{\beta+2}
$$

Note that the functional form of $h(n)$ is the same for gravitational waves and density perturbations, the difference is in the numerical coefficient (omitted in this discussion) which is somewhat in favor of gravitational waves [2,7]. The numerical level of $h(n)$ is mainly controlled by the constant $l_{o}$.

The spectra of other quantities can be found in the same manner. For instance, in case of density perturbations, one can derive the spectrum of perturbations in the matter density $\delta \rho / \rho$. Since the relationship between $\delta \rho / \rho$ and the metric perturbations involves the factor $(n \eta)^{2}$ and, hence, involves two extra powers of $n, \frac{\delta \rho}{\rho}(n) \sim n^{2} h(n)$, one finds

$$
\left\langle 0\left|\frac{\delta \rho}{\rho} \frac{\delta \rho}{\rho}\right| 0\right\rangle \sim \int_{0}^{\infty} P_{Z}^{\rho}(n) \frac{d n}{n}
$$

where $P_{Z}^{\rho}(n) \sim\left(l_{P l}^{2} / l_{o}^{2}\right) n^{2(\beta+4)}$ and

$$
\frac{\delta \rho}{\rho}(n) \sim \frac{l_{P l}}{l_{o}} n^{\beta+4} .
$$


It follows from Eq. (16) that $h(n)$ is independent of $n$ if $\beta=-2$. This independence corresponds to the original Zeldovich's definition of the "flat" spectrum: all waves enter the Hubble radius with the same amplitude. If the gravitational field perturbations $h(n)$, regardless of their wavelength, have the same amplitude upon entering the Hubble radius, the matter density perturbations $\frac{\delta \rho}{\rho}(n)$ do also have the same amplitude (the extra factor $(n \eta)^{2}$ is of the order of 1 at the time when a given wave $n$ enters the Hubble radius). For models of the very early Universe governed by a scalar field, the spectral index $\beta+2$ in Eq. (16) can never be positive.

Formula (16) and the associated formula (17) should be compared with the "standard" inflationary formula which requires that the amplitudes of density perturbations taken at the time of entering the Hubble radius should go to infinity in the limit of the De Sitter inflation, $\beta \rightarrow-2$. It should also be noted that a "disgusting convention" (the term is borrowed from Ref. [9]) is often being used according to which one and the same HarrisonZeldovich spectrum is described by the spectral index $n_{t}=0$ for gravitational waves and by the spectral index $n_{s}=1$ for density perturbations. Of course, there is no need in this convention. In both cases, the metric perturbations with the Harrison-Zeldovich spectrum are described by the same spectral index (zero), see Eq. (16).

We will finish this section with a short discussion of coherent states. There is no natural mechanism for the generation of cosmological perturbations in coherent states, but if there were one it would be reflected in many parts of the theory. The interaction part of the Hamiltonian (6) would be linear (not quadratic) in the creation and annihilation operators. The analogue of Eq. (7) would read

$$
\begin{aligned}
& c_{\mathbf{n}}(\eta)=e^{-i n \eta} c_{\mathbf{n}}(0)+\alpha_{n}(\eta) \\
& c_{\mathbf{n}}^{\dagger}(\eta)=e^{i n \eta} c_{\mathbf{n}}^{\dagger}(0)+\alpha_{n}^{*}(\eta),
\end{aligned}
$$

where the complex function $\alpha_{n}(\eta)$ is determined by the coupling function in the Hamiltonian. On the position - momentum diagram, the evolution (18) of the field operators corresponds to displacing the vacuum state without squeezing while the evolution (7) corresponds to squeezing the vacuum state without displacing. In terminology of mechanics, coherent states are produced by a force acting on the oscillator while squeezed vacuum states are produced by a parametric influence. In the coherent states, the mean value of the field is not zero. The correlation functions would also be different what would eventually be reflected in the differing statistical properties of the field (at least, for some quantities). 


\section{QUANTUM-MECHANICAL EXPECTATION VALUES FOR THE MICROWAVE BACKGROUND ANISOTROPIES}

In absence of cosmological perturbations, the temperature of the microwave background radiation seen in all directions on the sky would be the same, $T$. Let us denote a direction on the sky by a unit vector e. The presence of cosmological perturbations makes the temperature seen in the direction e differing from $T$. The temperature perturbation produced by density perturbations or gravitational waves can be described by the formula [10]:

$$
\frac{\delta T}{T}(\mathbf{e})=\frac{1}{2} \int_{0}^{w_{1}} \frac{\partial h_{i j}}{\partial \eta} e^{i} e^{j} d w
$$

where $\partial h_{i j} / \partial \eta$ is taken along the integration path $x^{i}=e^{i} w, \eta=\eta_{R}-w$, from the event of reception $w=0$ to the event of emission $w=w_{1}=\eta_{R}-\eta_{E}$. The formula for rotational perturbations is more complicated than (19) [10], and we will leave rotational perturbations aside.

For quantized cosmological perturbations, the $\frac{\delta T}{T}(\mathbf{e})$ becomes a quantum-mechanical operator. Using Eq. (11) we can write this operator as

$$
\begin{aligned}
& \frac{\delta T}{T}(\mathbf{e})=\frac{C}{2} \frac{1}{(2 \pi)^{3 / 2}} \int_{0}^{w_{1}} d w \int_{-\infty}^{\infty} d^{3} n \sum_{s=1}^{2} \stackrel{s}{p}_{i j}(\mathbf{n}) e^{i} e^{j}
\end{aligned}
$$

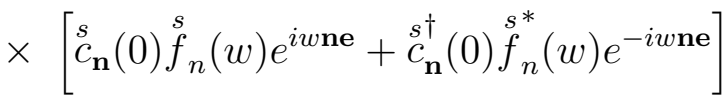

where

$$
\left.\stackrel{s}{f_{n}}(w) \equiv \frac{1}{\sqrt{2 n}} \frac{d h_{n}}{d \eta}\right|_{\eta=\eta_{R}-w} .
$$

Having defined the observable $\frac{\delta T}{T}(\mathbf{e})$ and knowing the quantum state $|0\rangle$ we can compute various quantum-mechanical expectation values. In the laboratory quantum mechanics, the verification of theoretical predictions expressed in terms of the expectation values would require experiments on many identical systems. An immediate generalization of this principle to cosmology would require speculations about outcomes of experiments performed in "many identical universes". Without having access to "many universes" we can only rely on the mean (expected) values of the observables and on the probability distribution functions as indicators of what is likely or not to be observed in our own single Universe. We will return to this point in Sec. IV.

The expected value of the temperature perturbation to be observed in every fixed direction on the sky is zero:

$$
\left\langle 0\left|\frac{\delta T}{T}(\mathbf{e})\right| 0\right\rangle=0
$$

One particular measured temperature map is the result of the measurement performed over one particular realization of the random process describing cosmological perturbations of quantum-mechanical origin. For this realization, the temperature perturbations may, 
should, and in fact are, present. Many measurements will not help (except of reducing the instrumental noises) in the sense that they all should give identical results, because the timescale of the perturbations under discussion is so enormously larger than an interval of time between the experiments. If the COBE's map is correct, we will have to live with this map practically forever.

Let us now compute the expected angular correlation function for the temperature perturbations seen in two given directions on the sky, $\mathbf{e}_{1}$ and $\mathbf{e}_{2}$. This correlation function is defined as the mean value for the product of $\frac{\delta T}{T}\left(\mathbf{e}_{1}\right)$ and $\frac{\delta T}{T}\left(\mathbf{e}_{2}\right)$ :

$$
K\left(\mathbf{e}_{1}, \mathbf{e}_{2}\right)=\left\langle 0\left|\frac{\delta T}{T}\left(\mathbf{e}_{1}\right) \frac{\delta T}{T}\left(\mathbf{e}_{2}\right)\right| 0\right\rangle
$$

By manipulating with the product of two expressions (20) one can derive the formula

$$
\begin{aligned}
K\left(\mathbf{e}_{1}, \mathbf{e}_{2}\right)= & \frac{1}{4} C^{2} \frac{1}{(2 \pi)^{3}} \int_{0}^{w_{1}} d w \int_{0}^{w_{1}} d \bar{w} \int_{-\infty}^{\infty} d^{3} n e^{i \mathbf{n}\left(\mathbf{e}_{1} w-\mathbf{e}_{2} \bar{w}\right)} \\
& \times \sum_{s=1}^{2}\left(\stackrel{s}{p}_{i j}(\mathbf{n}) e_{1}^{i} e_{1}^{j}\right)\left(\stackrel{s}{p}_{i j}(\mathbf{n}) e_{2}^{i} e_{2}^{j}\right) \stackrel{s}{f_{n}}(w) \stackrel{s}{f}_{n}^{*}(\bar{w})
\end{aligned}
$$

The next step is the formidable task of taking the integrals over angular variables in 3dimensional wave-vector $\mathbf{n}$ space. However, it can be done (see Ref. [11] for gravitational waves, Ref. [12] for rotational perturbations, and Ref. [2] for density perturbations). The final expression reduces, without making any additional assumptions whatsoever, to the form

$$
K\left(\mathbf{e}_{1}, \mathbf{e}_{2}\right)=K(\delta)=l_{P l}^{2} \sum_{l=l_{\min }}^{\infty} K_{l} P_{l}(\cos \delta)
$$

We see that the correlation function depends only on the angle $\delta$ between the directions $\mathbf{e}_{1}, \mathbf{e}_{2}$ not directions themselves. The coefficient $l_{P l}^{2}$ is taken from $C^{2}$, other numerical coefficients are included in $K_{l}$. The quantities $K_{l}$ involve the integration of $\stackrel{s}{f}_{n}(w)$ over the parameter $w$ and the remaining integration over the wave-numbers $n$. The numerical values of $K_{l}$ depend on a chosen sort of cosmological perturbations and a chosen cosmological model; so far, the formula $(23)$ is totally general. $P_{l}(\cos \delta)$ are the Legendre polynomials. The lowest multipole $l_{\min }$ follows automatically from the theory and it turns out to be, not surprisingly, $l_{\min }=0$ for density perturbations, $l_{\min }=2$ for gravitational waves (and $l_{\min }=1$ for rotational perturbations). For the separation angle $\delta=0$, Eq. (23) reduces to the variance of $\frac{\delta T}{T}(\mathbf{e})$, that is

$$
\left\langle 0\left|\frac{\delta T}{T}(\mathbf{e}) \frac{\delta T}{T}(\mathbf{e})\right| 0\right\rangle=K(0)=l_{P l}^{2} \sum_{l=l_{\min }}^{\infty} K_{l} .
$$

Formula (23) gives the expected value of the observable $\frac{\delta T}{T}\left(\mathbf{e}_{1}\right) \frac{\delta T}{T}\left(\mathbf{e}_{2}\right)$. If the experimenter measured this observable in "many universes" and averaged the measured numbers, he/she would get the result (23). Moreover, formula (23) says that if the experimenter made the measurements at any other pair of directions, but with the same separation angle $\delta$, he/she would again get, after the averaging over "many universes", the result (23). Without having 
access to "many universes", we can ask what is the theoretical standard deviation of the quantity $\frac{\delta T}{T}\left(\mathbf{e}_{1}\right) \frac{\delta T}{T}\left(\mathbf{e}_{2}\right)$. (In practice, for deriving $K(\delta)$ we need a kind of ergodic hypothesis allowing to replace the averaging over "universes" by the averaging over pixels separated by a given angle on a single map.) The variance $V\left(\mathbf{e}_{1}, \mathbf{e}_{2}\right)$ of this quantity is, by definition,

$$
V\left(\mathbf{e}_{1}, \mathbf{e}_{2}\right)=\left\langle 0\left|\frac{\delta T}{T}\left(\mathbf{e}_{1}\right) \frac{\delta T}{T}\left(\mathbf{e}_{2}\right) \frac{\delta T}{T}\left(\mathbf{e}_{1}\right) \frac{\delta T}{T}\left(\mathbf{e}_{2}\right)\right| 0\right\rangle-\left[\left\langle 0\left|\frac{\delta T}{T}\left(\mathbf{e}_{1}\right) \frac{\delta T}{T}\left(\mathbf{e}_{2}\right)\right| 0\right\rangle\right]^{2} .
$$

The standard deviation is the square root of this number.

The calculation of $V\left(\mathbf{e}_{1}, \mathbf{e}_{2}\right)$ requires us to deal with the product of four expressions (20). However, the mean values of the products of four creation and annihilation operators are easy to handle. One can show that $V\left(\mathbf{e}_{1}, \mathbf{e}_{2}\right)$ depends only on the separation angle $\delta$ and

$$
V\left(\mathbf{e}_{1}, \mathbf{e}_{2}\right)=V(\delta)=\left[\left\langle 0\left|\frac{\delta T}{T}\left(\mathbf{e}_{1}\right) \frac{\delta T}{T}\left(\mathbf{e}_{2}\right)\right| 0\right\rangle\right]^{2}+\left[\left\langle 0\left|\frac{\delta T}{T}(\mathbf{e}) \frac{\delta T}{T}(\mathbf{e})\right| 0\right\rangle\right]^{2},
$$

that is

$$
V(\delta)=K^{2}(\delta)+K^{2}(0)
$$

The standard deviation for the observable $\frac{\delta T}{T}\left(\mathbf{e}_{1}\right) \frac{\delta T}{T}\left(\mathbf{e}_{2}\right)$ is

$$
\sigma(\delta)=[V(\delta)]^{1 / 2}=\sqrt{K^{2}(\delta)+K^{2}(0)} .
$$

In a similar fashion one can derive the higher order correlation functions for two directions $\mathbf{e}_{1}, \mathbf{e}_{2}$ and the correlation functions for larger number of directions, but we will not need this information.

In the limit $\delta=0$ Eqs. (25), (26) say that

$$
\left\langle 0\left|\left[\frac{\delta T}{T}(\mathbf{e})\right]^{4}\right| 0\right\rangle=3\left[\left\langle 0\left|\left[\frac{\delta T}{T}(\mathbf{e})\right]^{2}\right| 0\right\rangle\right]^{2} .
$$

The familiar factor 3 relating the fourth-order moment with the square of the second-order moment (given that the first-order moment is equal to zero) is the reflection of the underlying Gaussian nature of the squeezed vacuum wavefunctions associated with the Hamiltonian (6).

By examining Eq. (28) one can conclude that for each separation angle $\delta$ the standard deviation of the angular correlation function is very big. Even at those separation angles at which $K(\delta)$ vanishes, the standard deviation is as big as the variance for $\frac{\delta T}{T}(\mathbf{e})$ itself. However, the value of the standard deviation for a given variable is not very informative per se, as long as the probability density function for this variable is not known. If the probability density function (p.d.f.) were normal, we could say that the probability to find a result outside of $1 \sigma$ interval is $32 \%$. Without knowing the p.d.f. we could resort to the Chebyshev inequality, but it would only tell us that this probability is less than 1 . To get more information about possible deviation of the angular correlation function from its mean values we will consider in Sec. IV a classical random model which will reproduce the expectation values calculated above and will allow us to construct the p.d.f. for the variable $\frac{\delta T}{T}\left(\mathbf{e}_{1}\right) \frac{\delta T}{T}\left(\mathbf{e}_{2}\right)$. On the other hand, the quantum-mechanical calculations of this Section will shed light on the classical model. As is known, "quantum mechanics helps us understand classical mechanics", see on this subject a paper of Zeldovich signed by the pseudonym Paradoksov [13]. 


\section{CLASSICAL MODEL FOR THE STATISTICS OF THE MICROWAVE BACKGROUND ANISOTROPIES}

A distribution of the microwave background temperature over the sky is a real function of the angular coordinates. Assuming that $\delta T / T$ is a sufficiently smooth function on a sphere, one can expand it over the set of orthonormal complex spherical harmonics $Y_{l m}(\theta, \phi)[14]$ :

$$
\frac{\delta T}{T}(\mathbf{e})=\sum_{l=0}^{\infty} \sum_{m=-l}^{l}\left[a_{l m} Y_{l m}(\mathbf{e})+a_{l m}^{*} Y_{l m}^{*}(\mathbf{e})\right] .
$$

We want to formulate a statistical hypothesis about the coefficients $a_{l m}$, so it is better to write them first in terms of real $(r)$ and imaginary $(i)$ components:

$$
\begin{array}{rlrl}
a_{l m} & =a_{l m}^{r}+i a_{l m}^{i}, & a_{l m}^{*} & =a_{l m}^{r}-i a_{l m}^{i} \\
Y_{l m} & =Y_{l m}^{r}+i Y_{l m}^{i}, & Y_{l m}^{*} & =Y_{l m}^{r}-i Y_{l m}^{i}, \\
\frac{\delta T}{T}(\mathbf{e}) & =2 \sum_{l=0}^{\infty} \sum_{m=-l}^{l}\left[a_{l m}^{r} Y_{l m}^{r}(\mathbf{e})-a_{l m}^{i} Y_{l m}^{i}(\mathbf{e})\right] .
\end{array}
$$

Our statistical hypothesis is as follows: (i) all members of the set of random variables $\left\{a_{l m}^{r}, a_{l m}^{i}\right\}$ are statistically independent, (ii) each individual variable is normally distributed and has a zero mean, (iii) all variables with the same index $l$ have the same standard deviation $\sigma_{l}$. All said is expressed by the probability density function (p.d.f.) for individual variables:

$$
f\left(a_{l m}^{r}\right)=\frac{1}{\sqrt{2 \pi} \sigma_{l}} e^{-\frac{\left(a_{l m}^{r}\right)^{2}}{2 \sigma_{l}^{2}}}, \quad f\left(a_{l m}^{i}\right)=\frac{1}{\sqrt{2 \pi} \sigma_{l}} e^{-\frac{\left(a_{l m}^{i}\right)^{2}}{2 \sigma_{l}^{2}}},
$$

and by the p.d.f. for the entire set of variables, which is simply a product of all p.d.f.'s for all individual variables:

$$
f\left(\left\{a_{l m}^{r}, a_{l m}^{i}\right\}\right)=\sqcap_{l, m} f\left(a_{l m}^{r}\right) f\left(a_{l m}^{i}\right) .
$$

Having postulated the p.d.f.'s, we can now compute the expectation values of certain functions of the random variables. Below, the angular brackets will denote the expectation values calculated with the help of the p.d.f. (33), unless other definition is stated.

Obviously, all linear functions have a zero mean:

$$
<a_{l m}^{r}>=0, \quad<a_{l m}^{i}>=0
$$

For quadratic combinations we have

$$
\begin{array}{ll}
<a_{l_{1} m_{1}}^{r} a_{l_{2} m_{2}}^{r}>=\sigma_{l_{1}}^{2} \delta_{l_{1} l_{2}} \delta_{m_{1} m_{2}}, & <a_{l_{1} m_{1}}^{i} a_{l_{2} m_{2}}^{i}>=\sigma_{l_{1}}^{2} \delta_{l_{1} l_{2}} \delta_{m_{1} m_{2}} \\
<a_{l_{1} m_{1}}^{r} a_{l_{2} m_{2}}^{i}>=0, & <a_{l_{1} m_{1}}^{i} a_{l_{2} m_{2}}^{r}>=0
\end{array}
$$

All triple products have zero means. Among quartic combinations, only those can survive which have four indices $(r)$, or four indices $(i)$, or two indices $(r)$ and two indices $(i)$. Two representative expressions are: 


$$
\begin{aligned}
& <a_{l_{1} m_{1}}^{r} a_{l_{2} m_{2}}^{r} a_{l_{3} m_{3}}^{r} a_{l_{4} m_{4}}^{r}>=\sigma_{l_{1}}^{2} \sigma_{l_{3}}^{2} \delta_{l_{1} l_{2}} \delta_{m_{1} m_{2}} \delta_{l_{3} l_{4}} \delta_{m_{3} m_{4}} \\
+ & \sigma_{l_{1}}^{2} \sigma_{l_{1}}^{2} \delta_{l_{1} l_{3}} \delta_{m_{1} m_{3}} \delta_{l_{2} l_{4}} \delta_{m_{2} m_{4}}+\sigma_{l_{1}}^{2} \sigma_{l_{2}}^{2} \delta_{l_{l_{4}} l_{4}} \delta_{m_{1} m_{4}} \delta_{l_{2} l_{3}} \delta_{m_{2} m_{3}} \\
< & <a_{l_{1} m_{1}}^{r} a_{l_{2} m_{2}}^{r} a_{l_{3} m_{3}}^{i} a_{l_{4} m_{4}}^{i}>=\sigma_{l_{1}}^{2} \sigma_{l_{3}}^{2} \delta_{l_{1} l_{2}} \delta_{m_{1} m_{2}} \delta_{l_{3} l_{4}} \delta_{m_{3} m_{4}}
\end{aligned}
$$

Other quartic combinations can be obtained by the replacement $(r) \leftrightarrow(i)$ in Eqs. (36), (37) (or by permutation of pairs $(l m)$ in case of Eq. (37)). The higher-order correlations can be derived in a similar way, but we will not need them.

In our further calculations related to the random variables $\frac{\delta T}{T}(\mathbf{e})$ and $\frac{\delta T}{T}\left(\mathbf{e}_{1}\right) \frac{\delta T}{T}\left(\mathbf{e}_{2}\right)$ it is easier to deal with the complex coefficients $a_{l m}$, so we will first translate the above relationships to them. By using the available information one can derive

$$
\begin{aligned}
<a_{l m}> & =0, \quad<a_{l_{1} m_{1}} a_{l_{2} m_{2}}^{*}>=2 \sigma_{l}^{2} \delta_{l_{1} l_{2}} \delta_{m_{1} m_{2}} \\
<a_{l_{1} m_{1}} a_{l_{2} m_{2}} a_{l_{3} m_{3}}^{*} a_{l_{4} m_{4}}^{*}> & =4 \sigma_{l_{1}}^{2} \sigma_{l_{2}}^{2}\left(\delta_{l_{1} l_{3}} \delta_{m_{1} m_{3}} \delta_{l_{2} l_{4}} \delta_{m_{2} m_{4}}+\delta_{l_{1} l_{4}} \delta_{m_{1} m_{4}} \delta_{l_{2} l_{3}} \delta_{m_{3} m_{3}}\right) .
\end{aligned}
$$

The mean values of the complex conjugated quantities are given by the same formulas (38). Other nonvanishing quartic combinations can be obtained from the one in Eq. (38) by the permutation of pairs $(l m)$.

Now, even before deriving the p.d.f.'s for the random variables $\frac{\delta T}{T}(\mathbf{e})$ and $\frac{\delta T}{T}\left(\mathbf{e}_{1}\right) \frac{\delta T}{T}\left(\mathbf{e}_{2}\right)$, we can find some expectation values. It is clear from the definition (30) and Eq. (38) that

$$
\left\langle\frac{\delta T}{T}(\mathbf{e})\right\rangle=0
$$

When calculating the angular correlation function one should remember that

$$
\sum_{m=-l}^{l} Y_{l m}\left(\mathbf{e}_{1}\right) Y_{l m}^{*}\left(\mathbf{e}_{2}\right)=\frac{2 l+1}{4 \pi} P_{l}(\cos \delta)
$$

(note the origin of the factor $2 l+1$ which will accompany us often). By taking the product of two expressions (30) and using Eqs. (38), (40) one can find the angular correlation function

$$
\left\langle\frac{\delta T}{T}\left(\mathbf{e}_{1}\right) \frac{\delta T}{T}\left(\mathbf{e}_{2}\right)\right\rangle=\frac{1}{\pi} \sum_{l=0}^{\infty} \sigma_{l}^{2}(2 l+1) P_{l}(\cos \delta) .
$$

If the separation angle $\delta$ is zero, we obtain

$$
\left\langle\frac{\delta T}{T}(\mathbf{e}) \frac{\delta T}{T}(\mathbf{e})\right\rangle=\frac{1}{\pi} \sum_{l=0}^{\infty} \sigma_{l}^{2}(2 l+1) .
$$

[One may notice an incidental fact that the mean value of the random variable $a_{l}^{2}$ defined as $a_{l}^{2}=\sum_{m=-l}^{l} a_{l m} a_{l m}^{*}$ is $\left\langle a_{l}^{2}\right\rangle=2(2 l+1) \sigma_{l}^{2}$, that is the same expression which enters Eq. (41). This may suggest an interpretation of the quantity $\left\langle a_{l}^{4}\right\rangle-\left\langle a_{l}^{2}\right\rangle^{2}$ as the variance of the multipole moments. It would be an error, see Appendix.]

We can also find the 4 th order expectation values. The product of 4 expressions (30) in conjunction with Eq. (38) gives 


$$
\begin{aligned}
& \left\langle\frac{\delta T}{T}\left(\mathbf{e}_{1}\right) \frac{\delta T}{T}\left(\mathbf{e}_{2}\right) \frac{\delta T}{T}\left(\mathbf{e}_{1}\right) \frac{\delta T}{T}\left(\mathbf{e}_{2}\right)\right\rangle-\left\langle\frac{\delta T}{T}\left(\mathbf{e}_{1}\right) \frac{\delta T}{T}\left(\mathbf{e}_{2}\right)\right\rangle^{2} \\
& =\left\langle\frac{\delta T}{T}\left(\mathbf{e}_{1}\right) \frac{\delta T}{T}\left(\mathbf{e}_{2}\right)\right\rangle^{2}+\left\langle\frac{\delta T}{T}(\mathbf{e}) \frac{\delta T}{T}(\mathbf{e})\right\rangle^{2} .
\end{aligned}
$$

If $\delta=0$, it follows from Eq. (43) that

$$
\left\langle\left[\frac{\delta T}{T}(\mathbf{e})\right]^{4}\right\rangle=3\left\langle\left[\frac{\delta T}{T}(\mathbf{e})\right]^{2}\right\rangle^{2} .
$$

Up to difference in the meaning of the angular brackets, the formulas (39), (43), (44) reproduce the analogous results of the previous Section. Moreover, from comparison of Eqs. (23), (24) with Eqs. (41), (42) we can relate the quantities $K_{l}$, derivable from a given cosmological model plus perturbations, with the abstract quantities $\sigma_{l}$.

We can now engage in our major enterprise - the construction of the p.d.f. for the random variable $v \equiv \frac{\delta T}{T}\left(\mathbf{e}_{1}\right) \frac{\delta T}{T}\left(\mathbf{e}_{2}\right)$. We will start from the p.d.f. for the random variable $z \equiv \frac{\delta T}{T}(\mathbf{e})$. When it is necessary to distinguish directions $\mathbf{e}_{1}$ and $\mathbf{e}_{2}$, we will use the notations $z_{1}$ and $z_{2}$.

The variable $z$ is a function of the variables $\left\{a_{l m}^{r}, a_{l m}^{i}\right\}$ whose p.d.f.'s are known, Eqs. (31), (32). There exist regular methods (see, for example, an excellent book [15]) allowing to derive rigorously the p.d.f. of a function. However, in our case that the function is linear and all p.d.f's are normal, we can partially rely on a guesswork. Combining formulas and guessing we can write

$$
f(z)=\frac{1}{\sqrt{2 \pi} \sigma_{z}} e^{-\frac{z^{2}}{2 \sigma_{z}^{2}}}
$$

where

$$
\sigma_{z}^{2}=\frac{1}{\pi} \sum_{l=0}^{\infty} \sigma_{l}^{2}(2 l+1)
$$

The p.d.f. (45) certainly leads to Eqs. (39), (42), (44). Moreover, it allows us to say that the probability to find $z$ outside of $1 \sigma_{z}$ interval is approximately $32 \%$ :

$$
P\left(|z|>\sigma_{z}\right) \approx 0.32
$$

We now introduce two variables, $z_{1}$ and $z_{2}$, and ask about the p.d.f. in the 2-dimensional space $\left(z_{1}, z_{2}\right)$. Again, partially relying on a guesswork, we find that

$$
f\left(z_{1}, z_{2}\right)=\frac{1}{2 \pi \sigma_{z}^{2} \sqrt{1-\rho^{2}}} \exp \left\{-\frac{1}{2 \sigma_{z}^{2}\left(1-\rho^{2}\right)}\left[z_{1}^{2}+z_{2}^{2}-2 \rho z_{1} z_{2}\right]\right\}
$$

where

$$
\rho \sigma_{z}^{2}=\frac{1}{\pi} \sum_{l=0}^{\infty}(2 l+1) \sigma_{l}^{2} P_{l}(\cos \delta), \quad|\rho| \leq 1
$$


(See Eq. (5.11.1) in Ref. [15]). First, we can check that the marginal distributions are correct. For $f\left(z_{1}\right)$, one obtains

$$
f\left(z_{1}\right)=\int_{-\infty}^{\infty} f\left(z_{1}, z_{2}\right) d z_{2}=\frac{1}{\sqrt{2 \pi} \sigma_{z}} e^{-\frac{z_{1}^{2}}{2 \sigma_{z}^{2}}}
$$

and one obtains a similar expression for $f\left(z_{2}\right)$. Second, one can check that

$$
\left\langle z_{1}^{2}\right\rangle=\sigma_{z}^{2}, \quad\left\langle z_{2}^{2}\right\rangle=\sigma_{z}^{2}, \quad\left\langle z_{1} z_{2}\right\rangle=\rho \sigma_{z}^{2}
$$

where the angular brackets mean the integration with the p.d.f. (47). These equalities are Eqs. (42), (41) which we must have obtained.

Finally, we shall derive the p.d.f. for the variable $v=z_{1} z_{2}$. We will do this in some detail following the prescriptions of [15].

Let us introduce the two new variables $\left(z_{1}, v\right)$ instead of $\left(z_{1}, z_{2}\right)$ according to the transformation

$$
z_{1}=z_{1}, \quad z_{2}=\frac{v}{z_{1}}
$$

The Jacobian of this transformation is $\mathcal{J}=1 / z_{1}$. The p.d.f. $f(v)$ is the result of the following integration:

$$
f(v)=\frac{1}{2 \pi \sigma_{z}^{2} \sqrt{1-\rho^{2}}} \int_{-\infty}^{\infty} \frac{1}{\left|z_{1}\right|} \exp \left\{-\frac{1}{2 \sigma_{z}^{2}\left(1-\rho^{2}\right)}\left[z_{1}^{2}+\frac{v^{2}}{z_{1}^{2}}-2 \rho v\right]\right\} d z_{1} .
$$

The integral over $z_{1}$ can be taken with the help of 3.471 .9 from [16]. The resulting p.d.f. can be written in the form:

$$
f(v)= \begin{cases}\frac{1}{\pi \sigma_{z}^{2} \sqrt{1-\rho^{2}}} e^{\frac{\rho v}{\sigma_{z}^{2}\left(1-\rho^{2}\right)}} K_{0}\left(\frac{v}{\sigma_{z}^{2}\left(1-\rho^{2}\right)}\right), & \text { for } v>0 \\ \frac{1}{\pi \sigma_{z}^{2} \sqrt{1-\rho^{2}}} e^{\frac{\rho v}{\sigma_{z}^{2}\left(1-\rho^{2}\right)}} K_{0}\left(\frac{-v}{\sigma_{z}^{2}\left(1-\rho^{2}\right)}\right), & \text { for } v<0\end{cases}
$$

where $K_{0}$ is the modified Bessel function of its argument [17].

The function $f(v)$ is quite complicated and the distribution is obviously not normal. The function $f(v)$ goes to zero for $v \rightarrow \pm \infty$ and diverges logarithmically at the point $v=0$. Even a verification of the normalization condition

$$
\int_{-\infty}^{\infty} f(v) d v=1
$$

is not trivial. However, with the help of $6.621 .3,9.131 .1,9.121 .7,1.624 .9$ and 1.623 .2 from [16] one can prove the validity of Eq. (50).

The mean value and the standard deviation of the variable $v$ are known, see Eqs. (41), $(43)$ :

$$
\langle v\rangle=\rho \sigma_{z}^{2}, \quad \sigma_{v}=\left[\left\langle v^{2}\right\rangle-\langle v\rangle^{2}\right]^{1 / 2}=\sigma_{z}^{2} \sqrt{\rho^{2}+1}
$$

We already knew that the standard deviation is big. We now see again that $\sigma_{v}=\sigma_{z}^{2}$ at the separation angles at which the angular correlation function vanishes, $\rho=0$, and $\sigma_{v}=\sqrt{2} \sigma_{z}^{2}$ 
at zero separation angle, $\rho=1$. For other separation angles, $\sigma_{v}$ lies between these two numbers.

Now that we know the p.d.f., we can assign probabilities to the different ranges of the variable $v$. For instance, we can calculate the probability that the measured $v$ will be found, say, outside of the $\lambda \sigma_{v}$ interval surrounding the mean value of $v$, where $\lambda$ is an arbitrary fixed number. The probability of our interest is

$$
P\left(|v-\langle v\rangle|>\lambda \sigma_{v}\right)=\int_{-\infty}^{\sigma_{z}^{2}\left(\rho-\lambda \sqrt{\rho^{2}+1}\right)} f(v) d v+\int_{\sigma_{z}^{2}\left(\rho+\lambda \sqrt{\rho^{2}+1}\right)}^{\infty} f(v) d v \quad .
$$

To get a qualitative estimate of the associated theoretical uncertainties for the observable $v$, we will ask a slightly different question. What should the number $\lambda$ be in order to have the 0.32 chance of finding $v$ outside the $\lambda \sigma_{v}$ interval and, hence, the 0.68 chance to find it inside the interval?

To evaluate the size of the disaster, we will start from the case $\rho=0$. In this case, the p.d.f. (49) is symmetric with respect to the origin $v=0$ (this is why $\langle v\rangle$ is zero in this case) and

$$
P\left(|v|>\lambda \sigma_{z}^{2}\right)=\frac{2}{\pi} \int_{\lambda}^{\infty} K_{0}(x) d x
$$

We want this number to be approximately equal to 0.32. Judging from the Fig. 9.7 in Ref. [17], a half of the area under the $K_{0}(x)$ function is accumulated when integrating from approximately $x=1 / 2$ and up to infinity. This means that $\lambda$ should approximately be equal to $1 / 2$.

If $\rho \neq 0$ the evaluation of $P$ is more complicated. For $\rho \neq 0$, the function (49) is not symmetric with respect to the origin $v=0$. It has larger values at positive $v$ 's if $\rho>0$ (this is why $\langle v\rangle>0$ in this case) and it has larger values at negative $v$ 's if $\rho<0$ (this is why $\langle v\rangle<0$ in this case). The graph of the function $e^{x} K_{0}(x)$ plotted on Fig. 9.8 in Ref. [17] is helpful. A qualitative analysis shows again that $\lambda$ is approximately equal to $1 / 2$. (More accurate estimates can of course be reached by numerical methods.)

At any rate, the $\frac{1}{2} \sigma_{v}$ interval gives approximately the same probability estimates as if the distribution (49) were normal. 


\section{CONCLUSIONS}

A particular cosmological model plus perturbations gives unambiguous predictions with regard to the expectation values of the measurable quantities. Differing models give different predictions. We want to distinguish them observationally and to learn about physics of the very early Universe. However, the quantum-mechanical origin of the cosmological perturbations is reflected in the theoretical statistical uncertainties surrounding the expectation values. One important measurable quantity is the angular correlation function of the microwave background anisotropies. Its mean value at the zero separation angle was denoted

$\sigma_{z}^{2}$ in this paper. It was shown that the standard deviation for the correlation function is very big. The $68 \%$ confidence level corresponds, approximately, to $\frac{1}{2} \sigma_{z}^{2}$ at the separation angles where the correlation function vanishes, to $\frac{\sqrt{2}}{2} \sigma_{z}^{2}$ at the zero separation angle, and to intermediate numbers for other separation angles.

The angular correlation function has actually been measured. It is presented at Fig. 3 in the paper [18]. The authors surround the measured points by a narrow shaded region which they address as follows: "The shaded region is the $68 \%$ confidence region .... including cosmic variance and instrument noise". It is not quite clear what the authors of Ref. [18] (see also Ref. [19]) mean by "cosmic variance", but if they mean the theoretical statistical uncertainties, these uncertainties are significantly larger than what is plotted. According to the calculations presented above, the half-width of the shaded region should be approximately $600(\mu K)^{2}$ near the points where the correlation function vanishes and approximately $840(\mu K)^{2}$ near the point marking the zero separation angle.

The conclusion is a bit disappointing. Apparently, God is telling us something important about the very early Universe by exhibiting the microwave background anisotropies, but the channel of information is so noisy that it will be hard to understand the message. 


\section{APPENDIX}

The set of random variables $\left\{a_{l m}^{r}, a_{l m}^{i}\right\}$ defined by Eqs. (32), (33) lives its own independent life regardless of whether or not the variables are considered random coefficients in the expansion of some function over spherical harmonics. Being such, it allows introduction of new functions and calculation of their expectation values. One interesting variable is defined by the equation

$$
a_{l}^{2}=\sum_{m=-l}^{l} a_{l m} a_{l m}^{*}=\sum_{m=-l}^{l}\left|a_{l m}\right|^{2}=\sum_{m=-l}^{l}\left[\left(a_{l m}^{r}\right)^{2}+\left(a_{l m}^{i}\right)^{2}\right] .
$$

By using Eq. (38) one can calculate the expectation value of $a_{l}^{2}$ :

$$
\left\langle a_{l}^{2}\right\rangle=(2 l+1) 2 \sigma_{l}^{2}
$$

The factor $(2 l+1)$ reflects the number of independent "degrees of freedom" associated with the index $l$. One can also introduce the variable $a_{l}^{4}$ and calculate its expectation value:

$$
\left\langle a_{l}^{4}\right\rangle=(2 l+1)(l+1) 8 \sigma_{l}^{4}=\left\langle a_{l}^{2}\right\rangle^{2} \frac{2(l+1)}{2 l+1} .
$$

The difference $\left\langle a_{l}^{4}\right\rangle-\left\langle a_{l}^{2}\right\rangle^{2}$ is, by definition, the variance of the variable $a_{l}^{2}$. From Eqs. (56), (55) one finds

$$
\left\langle a_{l}^{4}\right\rangle-\left\langle a_{l}^{2}\right\rangle^{2}=\frac{1}{2 l+1}\left\langle a_{l}^{2}\right\rangle^{2}
$$

Now, we can notice that the angular correlation function (41) can be written in the form

$$
\left\langle\frac{\delta T}{T}\left(\mathbf{e}_{1}\right) \frac{\delta T}{T}\left(\mathbf{e}_{2}\right)\right\rangle=\frac{1}{2 \pi} \sum_{l=0}^{\infty}\left\langle a_{l}^{2}\right\rangle P_{l}(\cos \delta) \quad .
$$

On this ground, there may be a temptation (which is, in fact, followed by many including observers) to write the random variable $\frac{\delta T}{T}\left(\mathbf{e}_{1}\right) \frac{\delta T}{T}\left(\mathbf{e}_{2}\right)$ in the form

$$
\frac{\delta T}{T}\left(\mathbf{e}_{1}\right) \frac{\delta T}{T}\left(\mathbf{e}_{2}\right)=\frac{1}{2 \pi} \sum_{l=0}^{\infty} a_{l}^{2} P_{l}(\cos \delta)
$$

and to interpret Eq. (57) as the variance for the multipole moments of the correlation function. One should strongly resist to this temptation.

Let us show that the definition (59) is incorrect despite the fact that it gives correct expectation value (58). It follows from the definition (59) that

$$
\begin{aligned}
& \frac{\delta T}{T}\left(\mathbf{e}_{1}\right) \frac{\delta T}{T}\left(\mathbf{e}_{2}\right) \frac{\delta T}{T}\left(\mathbf{e}_{1}\right) \frac{\delta T}{T}\left(\mathbf{e}_{2}\right) \\
& =\frac{1}{4 \pi^{2}} \sum_{l=0}^{\infty} a_{l}^{4}\left[P_{l}(\cos \delta)\right]^{2}+\frac{1}{4 \pi^{2}} \sum_{l, l^{\prime}=0, l \neq l^{\prime}}^{\infty} a_{l}^{2} P_{l}(\cos \delta) a_{l^{\prime}}^{2} P_{l^{\prime}}(\cos \delta)
\end{aligned}
$$


Using (56), (58) and remembering that $a_{l}^{2}$ and $a_{l^{\prime}}^{2}$ are statistically independent for $l \neq l^{\prime}$, one can find the expectation value of the quantity (60):

$$
\begin{aligned}
& \left\langle\frac{\delta T}{T}\left(\mathbf{e}_{1}\right) \frac{\delta T}{T}\left(\mathbf{e}_{2}\right) \frac{\delta T}{T}\left(\mathbf{e}_{1}\right) \frac{\delta T}{T}\left(\mathbf{e}_{2}\right)\right\rangle \\
& =\frac{1}{4 \pi^{2}} \sum_{l=0}^{\infty}\left\langle a_{l}^{4}\right\rangle\left[P_{l}(\cos \delta)\right]^{2}+\frac{1}{4 \pi^{2}} \sum_{l, l^{\prime}=0, l \neq l^{\prime}}^{\infty}\left\langle a_{l}^{2}\right\rangle\left\langle a_{l^{\prime}}^{2}\right\rangle P_{l}(\cos \delta) P_{l^{\prime}}(\cos \delta) \\
& =\frac{1}{4 \pi^{2}} \sum_{l=0}^{\infty}\left\langle a_{l}^{4}\right\rangle\left[P_{l}(\cos \delta)\right]^{2}+\frac{1}{4 \pi^{2}}\left[\sum_{l=0}^{\infty}\left\langle a_{l}^{2}\right\rangle P_{l}(\cos \delta)\right]^{2}-\frac{1}{4 \pi^{2}} \sum_{l=0}^{\infty}\left\langle a_{l}^{2}\right\rangle^{2}\left[P_{l}(\cos \delta)\right]^{2} \\
& =\left\langle\frac{\delta T}{T}\left(\mathbf{e}_{1}\right) \frac{\delta T}{T}\left(\mathbf{e}_{2}\right)\right\rangle^{2}+\frac{1}{4 \pi^{2}} \sum_{l=0}^{\infty} \frac{1}{2 l+1}\left\langle a_{l}^{2}\right\rangle^{2}\left[P_{l}(\cos \delta)\right]^{2} .
\end{aligned}
$$

It follows from (61) that the variance of the variable $\frac{\delta T}{T}\left(\mathbf{e}_{1}\right) \frac{\delta T}{T}\left(\mathbf{e}_{2}\right)$ would read (if (59) were correct):

$$
\frac{1}{\pi^{2}} \sum_{l=0}^{\infty}(2 l+1) \sigma_{l}^{4}\left[P_{l}(\cos \delta)\right]^{2}
$$

This expression should be compared with the correct variance following from Eq. (43):

$$
\frac{1}{\pi^{2}}\left[\sum_{l=0}^{\infty}(2 l+1) \sigma_{l}^{2} P_{l}(\cos \delta)\right]^{2}+\frac{1}{\pi^{2}}\left[\sum_{l=0}^{\infty}(2 l+1) \sigma_{l}^{2}\right]^{2} .
$$

Formulas (62), (63) disagree even for $\delta=0$, and even in their first, $l=0$ term. This shows that the ad hoc definition (59) is incorrect. The correct definition of the random variable $\frac{\delta T}{T}\left(\mathbf{e}_{1}\right) \frac{\delta T}{T}\left(\mathbf{e}_{2}\right)$ is the one following from the definition (30) and which we have used in this paper. 


\section{ACKNOWLEDGMENTS}

I appreciate discussions with Yu. V. Sidorov at the early phase of this study. This work was supported by NASA grants NAGW 2902, 3874 and NSF grant 92-22902. 


\section{REFERENCES}

[1] G. F. Smoot, et al., Astrophys. J. 396, L1 (1992).

[2] L. P. Grishchuk, Phys. Rev. D 50, 7154 (1994).

[3] L. P. Grishchuk and Y. V. Sidorov, Phys. Rev. D 42, 3413 (1990); Class. Quantum Grav. 6, L161 (1989).

[4] Special issues: J. Mod. Opt. 34 (6,7) (1987); J. Opt. Soc. Am. 84, (10) (1987).

[5] Special issue: Physica Scripta (Eds. W. P. Schleich and S. M. Barnett) T 48 (1993).

[6] N. Deruelle and V. F. Mukhanov, "On matching Conditions for Cosmological Perturbations", gr-qc 9503050.

[7] L. P. Grishchuk. Cosmological Perturbations of Quantum-Mechanical Origin and Anisotropy of the Microwave Background Radiation. Report WUGRAV-94-11, gr-qc 9410025.

[8] J. M. Niedra and A. I. Janis, G.R.G. 15, 241 (1983).

[9] P. J. Steinhardt. Cosmology at the Crossroads. Invited talk at the Snowmass meeting (to be published), astro-ph 9502024.

[10] R. K. Sachs and A. M. Wolfe, Astrophys. J. 1, 73 (1967).

[11] L. P. Grishchuk, Phys. Rev. D 48, 3513 (1993).

[12] L. P. Grishchuk, Phys. Rev. D 48, 5581 (1993).

[13] P. Paradoksov, Uspekhi Fiz. Nauk, 89, 707 (1966) [Sov. Phys. Uspekhi, 9, 618 (1967)].

[14] G. A. Korn and T. M. Korn, Mathematical Handbook for Scientists and Engineers (McGraw-Hill Book Co., 1968).

[15] M. Fisz, Probability Theory and Mathematical Statistics (J. Wiley \& Sons, 1967).

[16] I. S. Gradshteyn and I. M. Ryzhik, Table of Integrals, Series, and Products (Academic Press, 1980).

[17] M. Abramowitz and I. A. Stegun, (eds), Handbook of Mathematical Functions (Dover Publishing, 1972).

[18] C. L. Bennett, et al., Astrophys. J. 436, 423 (1994).

[19] E. L. Wright, et al., Astrophys. J. 436, 443 (1994).

[20] For gravitational waves this formula is in fact known for long time. See Eq. (5b) in L. P. Grishchuk, Ann. N.Y. Acad. Sci. 302, 439 (1977) where the notational replacement $h(n)=A(n)$ should be made and where $A(n)$ should be interpreted as the final (generated) characteristic amplitude, $A^{2}(n) \sim B^{2}(n) n^{2(1+\beta)}$, while $B(n)$ should be interpreted as the initial (zero-point quantum) characteristic amplitude, $B(n) \sim n$, in accord with the scale factor, Eq. (15) above, in which the $\eta$-time grows from $-\infty$. 